\title{
Advancing the Patient EXperience (APEX) in COPD Registry: Study Design and Strengths
}

\author{
Barbara P. Yawn, MD, MSc, MSPH, FAAFP, Alan Kaplan, MD, CCFP (EM), FCFP, \\ Wilson D. Pace, MD, Janwillem W. H. Kocks, MD, PhD, Lakmini Bulathsinhala, MPH, \\ Victoria A. Carter, BSc, Ku-Lang Chang, MD, FAAFP, MRO, Chelsea L. Edwards, PhD, \\ Chester Fox, MD, FAAFP, Gabriela Gaona, MPH, Gokul Gopalan, MD, MPH, \\ MeiLan K. Han, MD, MSc, Maja Kruszyk, BEng, Chantal E. Le Lievre, BPH, \\ Cathy D. Mable, PhD, MBA, Barry Make, MD, Zoe K. Philip, MPH, Chris Price, LLB, \\ Amanda R. Ratigan, PhD, MPH, Asif Shaikh, MD, MPH, Neil Skolnik, MD, \\ Brooklyn Stanley, MSc, and David B. Price, FRCGP
}

The Advancing the Patient Experience (APEX) in Chronic Obstructive Pulmonary Disease (COPD) registry (https:// www.apexcopd.org/) is the first primary care health system-based COPD registry in the United States. While its ultimate goal is to improve the care of patients diagnosed with COPD, the registry is also designed to describe real-life experiences of people with COPD, track key outcomes longitudinally, and assess the effectiveness of interventions. It will retrospectively and prospectively collect information from 3000 patients enrolled in 5 health care organizations. Information will be obtained from electronic health records, and from extended annual and brief questionnaires completed by patients before clinic visits. Core variables to be collected into the APEX COPD registry were agreed on by Delphi consensus and fall into 3 domains: demographics, COPD monitoring, and treatment. Main strengths of the registry include: 1) its size and scope (in terms of patient numbers, geographic spread and use of multiple information sources including patient-reported information); 2) collection of variables which are clinically relevant and practical to collect within primary care; 3) use of electronic data capture systems to ensure high-quality data and minimization of data-entry requirements; 4) inclusion of clinical, database development, management and communication experts; 5) regular sharing of key findings, both at international/national congresses and in peer-reviewed publications; and 6) a robust organizational structure to ensure continuance of the registry, and that research outputs are ethical, relevant and continue to bring value to both patients and physicians. ( $\mathrm{J}$ Am Board Fam Med 2021;34:22-31.)

Keywords: Ambulatory Care, Chronic Obstructive Pulmonary Disease, Delivery of Health Care, Registries, Patient Reported Outcome Measures, Primary Health Care, Retrospective Studies, Surveys and Questionnaires

\section{Introduction}

In 2001, the Global Strategy for the Diagnosis, Management and Prevention of Chronic Obstructive

This article was externally peer reviewed.

Submitted 9 July 2020; revised 30 September 2020; accepted 30 September 2020.

From the University of Minnesota, Minneapolis, MN (BPY); COPD Foundation, Washington DC (BPY); Observational and Pragmatic Research Institute, Singapore, Singapore (AK, JWHK, DBP); ${ }^{4}$ Family Physician Airways Group of Canada, Stouffville, Canada (AK); University of Toronto, Toronto, Canada (AK); DARTNet Institute, Aurora, CO (WDP, CF, GGa, ZKP, ARR); University of Colorado, Denver, CO (WDP); General Practitioners Research Institute, Groningen, Netherlands (JWHK); Optimum Patient Care, Cambridge, UK (LB, VAC, CLE, MK, CELL, CP, BS, DBP); College of Medicine, University of Florida, Gainesville, FL (KLC); University at Buffalo, Buffalo, NY (CF); Boehringer Ingelheim, Ridgefield, CT (GGo, CDM, AS); University of Michigan, Ann Arbor, MI (MKH); Department of Medicine, National Jewish Health, Denver, CO (BM); Thomas Jefferson University, Jenkintown, PA (NS); Abington Jefferson Health, Jenkintown,
Lung Disease (GOLD) was first published, marking a new generation of evidence-based recommendations. ${ }^{1}$ These recommendations were designed to

PA (NS); Centre of Academic Primary Care, Division of Applied Health Sciences, University of Äberdeen, Aberdeen, UK (DBP).

Funding: APEX COPD is conducted by Optimum Patient Care (OPC) Global Limited, and co-funded by OPC Global Ltd and Boehringer Ingelheim Pharmaceuticals, Inc (BIPI). The author(s) meet criteria for authorship as recommended by the International Committee of Medical Journal Editors. The authors received no direct compensation related to the development of the manuscript.

Conflict of interest: BPY has served on COPD-related advisory boards for GlaxoSmithKline, AstraZeneca, Novartis, and Boehringer Ingelheim, and received COPD-related investigator-initiated research funds from GlaxoSmithKline, Boehringer Ingelheim, AstraZeneca, and Novartis. AK is a member of the advisory board of, or speakers bureau for, AstraZeneca, Behring, Boehringer Ingelheim, Covis, Grifols, GlaxoSmithKline, Merck Frosst, Novo Nordisk, Novartis, Pfizer, Purdue, Sanofi, Teva, and Trudel. WDP is on the advisory board for Mylan; stock 
improve on existing chronic obstructive pulmonary disease (COPD) management strategies, and to be a core resource for the care of people with COPD globally. The most recent update of the GOLD strategy document outlines criteria for COPD diagnosis (including spirometry), provides evidence-based guidance on COPD treatment according to symptom burden and exacerbation risk, and highlights the importance of correct inhaler technique and regular technique assessment. ${ }^{2}$ Despite this well-outlined management strategy, COPD remains under- and misdiagnosed, ${ }^{3-5}$ with significant underuse of spirometry to confirm the COPD diagnosis. ${ }^{6,7}$

A series of campaigns to increase awareness of COPD and best clinical practice in primary care have been conducted. ${ }^{6,8,9}$ Appropriately, the focus has been on primary care in recognition of the fact that, within the United States, $80 \%$ of patients diagnosed with COPD are managed by their family physician or general internist. ${ }^{10}$ Despite these efforts, many US family physicians and other primary care clinicians are unfamiliar with recent advances in COPD management and updates to the GOLD strategy document. ${ }^{7}$ Making a diagnosis of COPD based on clinical findings alone is not uncommon. Prescribed treatments are not always aligned with COPD management strategy recommendations. ${ }^{11,12}$ Furthermore, adherence to treatment is low, in terms of taking medication and using inhalers correctly, both of which negatively impact treatment outcomes. ${ }^{13}$ Each of these factors has cost, effectiveness, and safety implications. For example, a survey including 784 US-based primary care physicians showed highly varied treatment preferences for COPD, and a strong preference for inhaled corticosteroid use, regardless of exacerbation history or knowledge of low lung function, in

from Novo Nordisk, Pfizer, Novartis, Johnson \& Johnson, Stryker, Amgen, Gilead, and Sanofi. JWHK declares grants and personal fees from AstraZeneca, Boehringer Ingelheim, GlaxoSmithKline, and Novartis, and grants from Chiesi, Mundipharma and Teva. LB, VAC, CLE, MK, CELL, CP, and BS are employees of Optimum Patient Care, a cofounder of the APEX COPD initiative. GGa, ZKP, and ARR are employees of the DARTNet Institute and report no conflict of interest. GGo is a former employee of the company Boehringer Ingelheim which is a co-founder of the APEX COPD initiative, and current employee of Vertex Pharmaceuticals. CDM and AS are employees of Boehringer Ingelheim, a co-founder of the APEX COPD initiative. MKH reports consulting for Boehringer Ingelheim, GlaxoSmithKline, and AstraZeneca, and research support from Novartis and Sunovion. BM reports funding from the National Heart, Lung and Blood Institute for the COPDGene study; grants and medical advisory boards from Boehringer Ingelheim, GlaxoSmithKline, AstraZeneca, and contravention of GOLD recommendations. ${ }^{2,11}$ Furthermore, poor adherence to COPD medications has been associated with reduced effectiveness and poor outcomes, including mortality. ${ }^{14}$

Concerned about the growing prevalence of COPD, members of the Congressional COPD Caucus; the National Institutes of Health's National Heart, Lung, and Blood Institute; National Heart and Lung Institute; Centers for Disease Control and Prevention; and many COPD community and health care professional stakeholders collaborated to formulate The COPD National Action Plan (Plan). ${ }^{15}$ The Plan outlines 5 goals to improve the management of COPD, including a focus on patient empowerment and improvement of disease prevention diagnosis and treatment. The Plan also encourages collection, analysis, and dissemination of public health data, highlights the need to continue research and to translate national recommendations into research and public health care actions. ${ }^{15}$ The use of newer technologies such as electronic health records (EHRs) and patient reported information/patient reported outcomes (PRI/PRO) data collection, and incorporation of these data, into a registry may be one way to support implementation of the National Action Plan.

Registries are well-established tools to observe the course of disease, understand the impact of treatment variations and outcomes, and examine factors that can influence prognosis. ${ }^{16}$ Key features that should be included in a registry have been summarized by Nelson and colleagues (Table 1). ${ }^{17}$ Registries can be particularly suited to tracking the natural progression of COPD, where progression is slow, and trends can be easily hidden. Registries are also useful to describe care patterns, disparities in care delivery, and to assess effectiveness, safety, and quality of care. ${ }^{16}$ The main

Sunovian; personal fees for DSMB from Spiration and Shire/Baxalta; CME personal fees from WebMD, National Jewish Health, American College of Chest Physicians, Projects in Knowledge, Hybrid Communications, SPIRE Learning, Ultimate Medical Academy, Catamount Medical, Eastern Pulmonary Society, Catamount Medical Communications Medscape, Eastern VA Medical Center, Academy Continued Healthcare Learning, and Mt. Sinai Medical Center; royalties from Up-To-Date; medical advisory boards from Novartis, Phillips, Third Pole, Science 24/7, and Vernoa; grants from Pearl; outside the submitted work. NS is on advisory boards for AstraZeneca, Teva, Lilly, Boehringer Ingelheim, Sanofi, Janssen Pharmaceuticals, Intarcia, Mylan, and GlaxoSmithKline; Payment for lectures/speaking engagements from AstraZeneca and Boehringer Ingelheim; Research Support from Sanofi, AstraZeneca, Boehringer Ingelheim, and GlaxoSmithKline. DBP has board membership with Amgen, AstraZeneca, Boehringer Ingelheim, Chiesi, Circassia, Mylan, 


\begin{tabular}{|c|c|}
\hline Feature & Delivered by APEX COPD registry \\
\hline $\begin{array}{l}\text { Social network of patients and families encouraged to engage in } \\
\text { the patient's healthcare and supported by tools that enable } \\
\text { them to track their health outcomes and support self-care }\end{array}$ & $\begin{array}{l}\text { The APEX COPD registry provides rapid feedback of data to } \\
\text { both patients and physicians to encourage engagement, support } \\
\text { shared decision making, and facilitate self-management by } \\
\text { enabling patients to track their disease over time. }\end{array}$ \\
\hline $\begin{array}{l}\text { Collaborative network of clinical teams that can provide care } \\
\text { and who engage in a system providing longitudinal and } \\
\text { comparative data }\end{array}$ & $\begin{array}{l}\text { The APEX COPD registry comprises a collaborative network of } \\
\text { primary care physicians, nurses, physician assistants, specialists, } \\
\text { research scientists, and database experts to collect longitudinal, } \\
\text { comparative, clinically relevant, and individual-level data. }\end{array}$ \\
\hline $\begin{array}{l}\text { Sharing of power and responsibility among patients, clinicians, } \\
\text { and scientists for designing, governing and evaluating services } \\
\text { to improve and research }\end{array}$ & $\begin{array}{l}\text { The APEX COPD registry is overseen by } 5 \text { governing bodies to } \\
\text { ensure collection of clinically relevant data, and ethically-sound } \\
\text { research. }\end{array}$ \\
\hline $\begin{array}{l}\text { Digital collection and use of both clinical and patient-reported } \\
\text { outcomes to guide care and as a basis for improvement, } \\
\text { research, and public health policy }\end{array}$ & $\begin{array}{l}\text { The APEX COPD registry collects data digitally from EHR and } \\
\text { from patients in the form of on-line questionnaires and during } \\
\text { the office visit. }\end{array}$ \\
\hline $\begin{array}{l}\text { Demonstration of measurable improvement in individual and } \\
\text { public health outcomes through improved adherence to } \\
\text { current evidence and rigorous trials of new approaches }\end{array}$ & $\begin{array}{l}\text { The APEX COPD registry will track all relevant variables } \\
\text { longitudinally to assess improvement in individual and public } \\
\text { health outcomes. }\end{array}$ \\
\hline $\begin{array}{l}\text { Dissemination and translation of ideas and findings through } \\
\text { publication in peer-reviewed journals, presentations and } \\
\text { meetings, and outreach to patients, clinicians, researchers, } \\
\text { and health policy analysts }\end{array}$ & $\begin{array}{l}\text { The APEX COPD registry incorporates a team of communication } \\
\text { specialists to ensure timely dissemination of research both at } \\
\text { scientific/medical congresses and in peer-reviewed publications. }\end{array}$ \\
\hline
\end{tabular}

APEX, Advancing the Patient Experience; COPD, chronic obstructive pulmonary disease; EHR, electronic health record.

Reproduced from Nelson EC, Dixon-Woods M, Batalden PB, et al. Patient focused registries can improve health, care, and science. 2021:354:i3319, with permission from BMJ Publishing Group Ltd.

advantages of prospective, observational patient registries are the inclusion of "real-world" patients, with enhanced generalizability, and the ability to examine clinical questions for which a randomized controlled trial (RCT) is either impractical or unethical. ${ }^{16,18}$ Registries also have the potential to improve diagnostics and may be used as a rich source of data to inform treatment algorithms or clinical decision support systems. Although a few COPD registries and patient cohorts already exist (eg, COPDGene and the COPD Foundation Patient-Powered Research Network Registry), ${ }^{19,20}$ none are based in primary care.

Mundipharma, Novartis, Regeneron Pharmaceuticals, Sanofi Genzyme, Teva Pharmaceuticals, ThermoFisher; consultancy agreements with Amgen, AstraZeneca, Boehringer Ingelheim, Chiesi, GlaxoSmithKline, Mylan, Mundipharma, Novartis, Pfizer, Teva Pharmaceuticals, Theravance; grants and unrestricted funding for investigator-initiated studies (conducted through Observational and Pragmatic Research Institute Pte Ltd) from AstraZeneca, Boehringer Ingelheim, Chiesi, Circassia, Mylan, Mundipharma, Novartis, Pfizer, Regeneron Pharmaceuticals, Respiratory Effectiveness Group, Sanofi Genzyme, Teva Pharmaceuticals, Theravance, UK National Health Service; payment for lectures/speaking engagements from AstraZeneca, Boehringer Ingelheim, Chiesi, Cipla, GlaxoSmithKline, Kyorin, Mylan, Mundipharma, Novartis, Regeneron Pharmaceuticals, Sanofi Genzyme, Teva Pharmaceuticals; payment for the development of educational materials from Mundipharma, Novartis; payment for travel/accommodation/meeting expenses from
A well-designed registry can provide benefits for all key stakeholders involved in COPD management. ${ }^{16}$ Physicians promptly receive a real-world picture of disease presentation, treatment practices, and outcomes in a large number of patients. Adherence to evidence-based recommendations can be assessed. Patients and patient advocacy organizations benefit from an increased understanding of the natural history of disease and the impact of treatment. Payers can obtain detailed information on the cost effectiveness of procedures, devices, or pharmaceuticals in a large number of patients. ${ }^{16}$ Unfortunately, few registries have realized their full potential. Data flow back to participating centers is frequently delayed; many registries rely on manual data entry, which is costly

AstraZeneca, Boehringer Ingelheim, Mundipharma, Mylan, Novartis, ThermoFisher; funding for patient enrolment or completion of research from Novartis; stock/stock options from AKL Research and Development Ltd which produces phytopharmaceuticals; owns $74 \%$ of the social enterprise Optimum Patient Care Ltd (Australia and UK) and 74\% of Observational and Pragmatic Research Institute Pte Ltd (Singapore); and is peer reviewer for grant committees of the Efficacy and Mechanism Evaluation programme, and Health Technology Assessment. KLC and CF declare no conflict of interest.

Corresponding author: David B. Price, FRCGP, Academic Primary Care, Division of Applied Health Sciences, University of Aberdeen, Polwarth Building, Foresterhill, Aberdeen AB25 2ZD, UK (E-mail: dprice@opri.sg). 
Table 2. APEX COPD Registry Core and Research Projects

Description

Delphi consensus of core variables to capture in the APEX COPD registry

Demographic and clinical baseline characteristics of COPD patients based in US primary care

Variation in demographic and clinical characteristics of COPD patients managed in US primary care

Treatment patterns of COPD patients managed in US primary care

APEX, Advancing the Patient Experience; COPD, chronic obstructive pulmonary disease.

and prone to error; and patients usually do not have access to their own data, or have the opportunity to add data outside the structure of the patient-physician/clinician consultation. ${ }^{17}$

In answer to the call to action of the COPD National Action Plan, ${ }^{15}$ the Advancing the Patient Experience (APEX) COPD registry (https://www. apexcopd.org/) will provide a mechanism to standardize and store data on COPD patients seen in primary care in the United States. It will integrate clinically relevant data from existing sources (ie, EHRs) and from patients themselves (through use of questionnaires and information obtained during the office visit) to answer key research questions relating to COPD in primary care. The primary objectives of the APEX COPD registry are to describe and characterize the primary care COPD population in the United States; to compare the effectiveness (both clinical and cost) and safety of current COPD treatments; and to understand predictors of response to available COPD treatment options. Current APEX COPD registry research projects closely align with these aims and are summarized in Table 2. Secondary objectives of the registry are numerous reflecting the scope of this initiative and its potential not only to improve both family physician awareness and management of COPD, but also to answer key research questions relevant to physicians treating COPD patients in every day clinical practice (Table 3). The aim of this article is to describe the APEX COPD registry study design and to summarize its strengths.

\section{Study Design of the APEX COPD Registry Registry Design}

This registry is a US-based, primary care registry, supported by a multinational and multi-organizational team of experts. It has been designed to be an observational, primary care initiative which will retrospectively and prospectively collect EHR COPD variables (from December 2008 through December 2022), supplemented with PRI/PRO data and information gathered from COPD patients during primary care visits. Due to the real-life nature of the

\section{Table 3. Secondary Objectives of the APEX COPD Registry Initiative}

Improve quality of care, and primary care patient outcomes through structured COPD support and increased understanding of COPD management

Understand the clinical phenotypes of COPD that predict response to or appropriateness of inhaled treatments

Understand the current burden and minimize side effects of steroid exposure through use of appropriate treatments

Support the development of effective and efficient diagnostic routines and therapeutic principles

Assess the level of classification differences as defined by comparing clinician-diagnosed COPD at baseline against established guidelines' diagnostic criteria

Describe disease management patterns such as treatment changes over time (eg, step up, step down, and switches), as well as the reasons for changes and the effect of these changes on disease progression

Assess the impact of inhaler technique, inhaler type and lung function on disease management and severity (eg, exacerbations and CAT scores)

Describe factors associated with treatment choice at baseline and describe disease progression

Describe risk factors (eg, age, sex, smoking, BMI, occupation, family history, presence of comorbidities, socioeconomic status, quality of care, lung function, exacerbations) associated with disease progression, PROs, and healthcare utilization

Assess the occurrence of exacerbations and other conditions, including URTIs and seasonal variations

Assess biomarker data and estimate their predictive value for disease diagnosis, pheno- and endo-type characterization, response to treatment and disease progression

Identify patients who may be eligible for participation in future research studies

APEX, Advancing the Patient Experience; BMI, body mass index; CAT, COPD assessment test; COPD, chronic obstructive pulmonary disease; PRI/PRO, patient-reported information \& outcomes; URTI, upper respiratory tract infection. 
registry, the routine clinical care of patients will continue to be provided in accordance with the patient and his/her health care professionals at the mutually agreeable utilization level. It is estimated that patients with prior COPD diagnoses will have at least 1 clinic visit per year. Central ethics (Institutional Review Board) approval has been obtained from the American Academy of Family Physicians for most sites (Institutional Review Board reference number 19-349). One site has its own ethics approval boards. Secondary ethics approval will also be sought where sites require their own ethics approval board. The study is registered with ENCePP (study reference number EUPAS29401; http://www.encepp.eu/encepp/view Resource.htm?id=29348).

\section{Patient Population}

Three thousand patients with COPD will be enrolled into the registry, identified using health care system EHR data. Patients will be recruited from participating primary care sites in accordance with local regulatory/ethical requirements. Patients will be eligible to participate if they have a diagnostic code for COPD (or a COPD monitoring review code), including chronic bronchitis, emphysema, $\alpha 1$-antitrypsin deficiency and mixed COPD/asthma, before, or at, the enrolment consultation, and were aged $\geq 35$ years at the time of COPD diagnosis. Registry activities include clarifying COPD diagnoses to further understand current diagnostic approaches in US primary care. Patients will be excluded if they are currently participating in a COPD drug therapy clinical trial at time of enrolment, have a life expectancy $<12$ months from date of enrolment, have had an active cancer diagnosis in the past 3 years (excluding nonmelanoma skin cancer), and/or are receiving hospice care. Informed consent will be obtained from patients via an online portal to allow data sharing for ethically approved research purposes as well as recruitment for future studies.

\section{Sites}

Clinic sites within 5 or more health care organizations (HCOs) will be recruited (Figure 1). Current participating sites (HCOs) are located in Texas (Bandera Family Health Center), Ohio (Metro Health System), Colorado (Miramont Family Medicine), New York (Urban Family Practice), and North Carolina (Wilmington Health System). Participating sites will provide access to a limited EHR data set through a data use agreement of all patients with
COPD who do not specifically opt out of the project. During the registry consent process, some patients may opt out of all data sharing. The data in the registry will be populated directly from EHRs with supplementary point of care data populated by primary care clinicians (eg, physicians, nurse practitioners and physician assistants working in primary care) during routine clinical care. These clinicians will be provided with an information pack outlining the APEX COPD initiative, including an overview of the information patients may bring to visits, operational and technical support for data entry (if required).

\section{Data Collection and Management Data Collection}

The APEX COPD registry will contain information obtained from multiple sources including additional information on assessment (eg, data from EHRs, patients, and clinicians at time of visits). The EHR data look-back period will be up to 10 years from December 1, 2018 for clinic visits, observations (eg, social history), measurements (eg, blood pressure, laboratory values), and procedures (eg, spirometry, surgeries). Medications and conditions (eg, diagnoses) will not be limited in their look-back period but extend back as far as EHRs exist at each site for patients included in the registry. PRI/PRO data will be provided via patient surveys (ie, baseline, annual and preclinic visit questionnaires). These data will be updated after, and potentially during, each encounter and supplemented by clinician-reported data collected for visit activities not recorded in the EHR (eg, inhaler technique observation).

\section{Variables Collected}

Basic demographic and diagnostic variables collected in the APEX COPD registry were developed by Delphi consensus. ${ }^{21}$ These are subdivided into 3 main areas: demographics, disease monitoring, and treatment variables. Specific data to be collected includes information on diagnosis, COPD exacerbations, symptom burden, lung function, quality of life, comorbidities, smoking status/history, treatment specifics (including side-effects), inhaler management (for example, inhaler satisfaction, and patient education/self-management (Appendix Table 1). ${ }^{21}$ Much of this information is extracted from existing EHRs, but some must be manually entered (eg, point of care variables; see Appendix Table 1). Additional variables used to assess COPD control (eg, use of rescue medication, physical activity and sputum color) ${ }^{22}$ and lung 
Figure 1. US states (blue) in which sites have been identified for inclusion in Advancing the Patient Experience in chronic obstructive pulmonary disease.

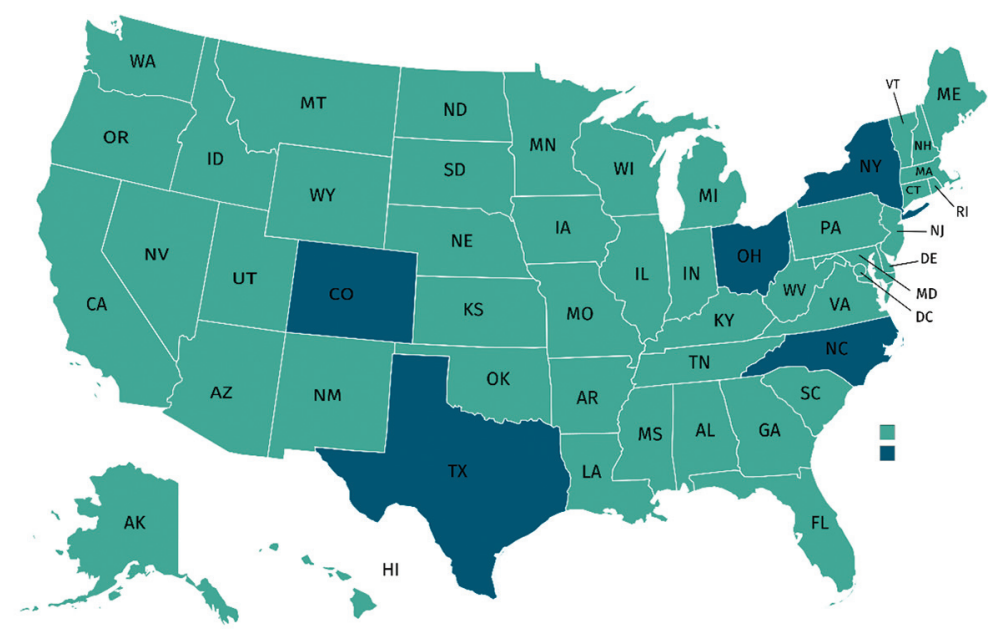

function were added to the registry after the Delphi exercise. These additional variables were agreed by consensus of the whole steering committee.

\section{Research Database}

High data quality standards will be maintained, and processes will be utilized to ensure that the data are as accurate as possible when presented for analysis. Data quality will be enhanced through a series of programmed data quality checks that automatically detect out of range or anomalous data. All modifications to data will be recorded in an audit log. EHR data will be extracted remotely by the DARTNet Institute (a nonprofit organization that hosts data sets of health information for quality improvement and research). Data will be standardized using the Observational Medical Outcomes Partnership common data model (v6), which allows for the analysis of data from disparate sources by transforming the data into a standard format and representation. The data will then be cleaned, quality checked, and deposited into a database on a biennial basis. PRI/PRO data will be collected into the Patient Engaged Electronic Reporting System (PEERS), a Health Insurance Portability and Accountability ACT (HIPAA) 1996compliant, Internet-based study management system; and paired with patient-level EHR data. To preserve confidentiality, all individuals will be assigned a

Figure 2. Operational overview of the Advancing the Patient Experience (APEX) in chronic obstructive pulmonary disease (COPD) initiative. Abbreviation: EHR, electronic health record.

(1)

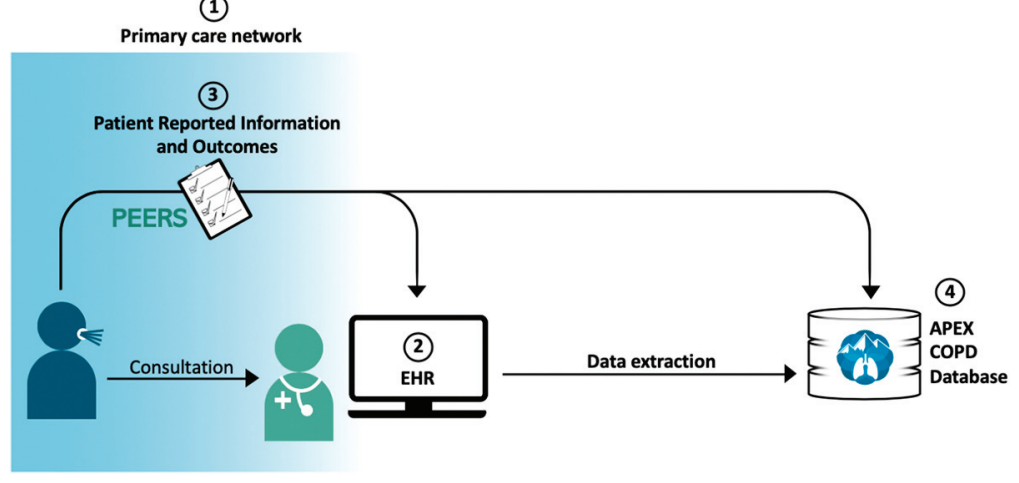


Figure 3. Key strengths of the Advancing the Patient Experience (APEX) in chronic obstructive pulmonary disease (COPD) initiative. Abbreviations: EHR, electronic health record; PoC, point of care; PRI/PRO, patient reported information/patient reported outcomes.

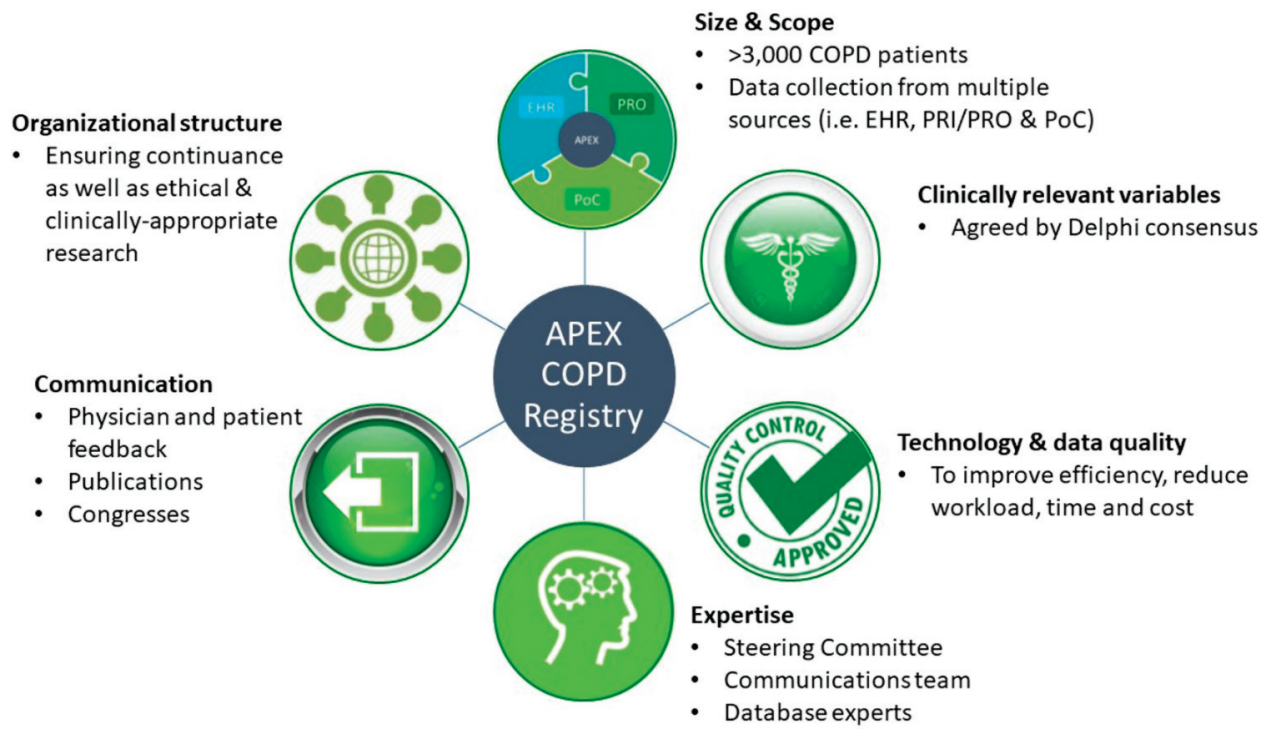

unique Registry ID using a 1-way hashing algorithm before being stored in the database. The APEX COPD database will be hosted in the United States on an Amazon Web Service (AWS) firewall-firewallprotected server. This server will be part of the HIPAA 1996-compliant DARTNet server environment maintained by AWS. Optimum Patient Care Global (OPC) will act as data custodians, but each site/patient will continue to own the patient-level data they contribute to the APEX COPD database.

\section{Research}

APEX COPD has a collaborative consensus voting system in place for the selection of research projects. Funded research proposals will be voted on annually by the APEX COPD Steering Committee. Each committee member has 1 vote. Voting is anonymous with a simple majority required for research project selection. All research projects will be approved by the US Steering Committee, the US Independent Review Board and the Anonymised Data Ethics \& Protocol Transparency (ADEPT) Committee. APEX COPD registry research proposals are summarized in Table 2.

\section{How It All Links Together: The Operational Delivery Model}

The APEX COPD registry incorporates 4 key and inter-related components (Figure 2). The primary care network includes a network of engaged clinicians and their patients diagnosed with COPD. The registry is fed by data obtained from existing EHRs and supplemented with PRI/PRO data (obtained via online questionnaires) and patient information gathered during office visits. All this information is gathered and extracted to the APEX COPD database. A clinical decision support system may be developed as an exploratory feature of the initiative in the future. This will provide current and evidence-based information support to primary care clinicians regarding treatment, monitoring, and care of COPD patients in primary care.

\section{Strengths of the APEX COPD Registry}

The strengths of the APEX COPD initiative and how it will improve management of COPD in primary care settings are summarized in Figure 3.

\section{Size and Scope}

This registry plans to capture information from 3000 COPD patients, from clinic sites within 4 to 5 HCOs, with wide geographic coverage throughout the United States (Figure 1). The scope of the initiative is broad, integrating information from multiple sources (ideally both EHR- and patient derived) to provide a snapshot of the COPD population managed in primary care in the United States; as 
well as the capability to conduct longitudinal analyses to examine the impact of appropriate COPD variables on disease prognosis, progression, and treatment outcomes (Table 1). The APEX COPD initiative, therefore, has the potential to improve primary care awareness of COPD and its management, and to answer key research questions, with the ultimate goal of improving care for patients diagnosed with COPD.

\section{Clinically Relevant Variables}

APEX COPD registry variables have been developed by consensus of the APEX COPD Delphi panel (Appendix Table 2). These variables will be integrated predominantly from existing EHRs ( $\mathrm{n}=115$ variables), but will also include up to 34 PRI/PRO variables and 5 variables collected from patients during office visits: forced expiratory volume in 1 second (forced vital capacity, moderate and severe exacerbations, and inhaler technique [Table 1; Appendix Table 1]). ${ }^{21}$ A balance was achieved between collecting the minimum information needed to effectively study the diagnosis and management of COPD, while also ensuring a comprehensive description of COPD patients managed in real-life clinical practice in the United States. Variable selection was also driven by practicality of collection by primary care clinicians, and the need to be clinically relevant and useful to physicians, other clinicians, and patients.

\section{Technology and Data Quality}

The true value of any registry is measured in terms of the quality of data it collects. Data provided for research will be analyzed as a limited data set at the individual patient level rather than the aggregate level. This offers the potential to explore clinically relevant and previously unanswered research questions, compared with the limited options possible with aggregate data. ${ }^{23}$ Electronic data capture systems will be used to capture data directly from EHR systems, which will improve efficiency, reduce workload, time, and cost, as well as enhance the quality of data collected, while minimizing data entry burden (Table 1).

\section{Clinical and Scientific Expertise}

Broad clinical expertise is integrated into each layer of the APEX COPD initiative. First, the APEX COPD Steering Committee consists of 14 experts in primary and specialist care from the United States and internationally (5 family physicians, 3 pulmonologists, and 6 respiratory researchers) with approximately $70 \%$ of members based in the United States (Appendix Table 2). These individuals are all experts in the field of COPD evidenced by their 1) publication history; 2) participation in the development and/or management of severe COPD registries, epidemiologic databases, and scientific congress committees and/or; 3) experience as a medical provider with interest in advancing COPD management in clinical practice. Second, primary care clinics involved in data acquisition will include experienced primary care doctors, nurse practitioners, and physician assistants. Lastly, the APEX COPD initiative includes an experienced communications team and partners with experts in database management and registry delivery (DARTNet Institute, Aurora, Colorado; Table 1).

\section{Communication}

The APEX COPD initiative plans to provide written feedback to both physicians and patients for use during clinic visits. Patients will receive feedback on meeting goals, health status, smoking and prevention, an inhaler satisfaction rating, and an annual treatment and hospitalization tracker. Physicians will receive feedback on patients' health test results (eg, GOLD category, COPD Assessment Test score) and information on smoking status, treatment adherence, and inhaler technique. The aim is to encourage patient empowerment, shared decision making, and improve physician-patient communication. APEX COPD registry research findings will also be regularly disseminated in peer-reviewed journals and at both national and international medical/scientific congresses (Table 1).

\section{Organizational Structure}

APEX COPD is overseen by 5 governing bodies: 1 ) OPC Global, 2) the Respiratory Effectiveness Group, 3) the ADEPT Committee, 4) the AAFP, and 5) the APEX Steering Committee. Their purpose is to safeguard the continuance of the registry into the future and to ensure that APEX COPD registry research is ethical, clinically appropriate, and continues to bring genuine value to patients and physicians (Table 1). The roles and responsibilities of each of these 5 governing bodies is provided in the Appendix. 


\section{Limitations and Advantages of Registry Data}

Certain limitations of registry data should be acknowledged. First, due to their design, registry data may possess lower internal validity than data collected prospectively in RCTs, limiting the extent to which they can support causal relationships. However, to counterbalance this limitation, the APEX COPD registry includes information from real-world patients receiving care they and their health care professionals direct, thus providing generalizable data not available from tightly controlled clinical trials with multiple exclusion criteria and limited enrolment settings. Second, the large volume of data collected by registries also creates the potential for many analyses with selective reporting. With the APEX COPD registry, this will be mitigated by a priori agreement of research protocols. A database is only as good as the data it collects, so a system to validate and verify data integrity (such as that employed by APEX COPD) is essential to ensure database utility. ${ }^{24}$ Lastly, although much of the information used to populate the APEX in COPD registry is extracted digitally from EHRs, some manual data entry is required both from healthcare professionals (HCPs) and patients, in the form of point of care and questionnaire variables, respectively. Minimization of the number of manual entry variables $(n=5)$ and use of shortened online patient questionnaires will reduce this burden. Administrative burden is also reduced as the Ethics Committees at each of the 5 sites have approved these questionnaires for implementation as part of clinical care. They do not require research consent. Furthermore, the APEX COPD registry will also supplement EHR data with information routinely recorded, such as inhaler technique education. ${ }^{25}$ Registry data may be collected prospectively and retrospectively using data that are routinely collected with opportunities to obtain more data, more quickly, over a long period of time, and at a lower cost than RCTs. ${ }^{25}$

\section{Conclusions}

The APEX COPD registry is the first health-system based primary care COPD registry in the United States, and closely aligns with the key features of a registry-based learning system. ${ }^{17}$ It will encourage patient engagement in care and shared decision making, use a collaborative network of clinical teams to provide longitudinal and comparative real-world care and outcomes data, conduct clinically relevant and ethical research, collect information from both physicians and patients, and is committed to dissemination of its findings to key stakeholders involved in the management of COPD in primary care. The APEX COPD registry will continue to evolve to best serve the needs of those living with and caring for people with COPD.

Writing, editorial support, and/or formatting assistance was provided by Ms. Audrey Ang of the Observational and Pragmatic Research Institute, Singapore; and Dr. Lisa Buttle of Medscript Ltd, Ireland, which was funded by BIPI. BIPI was given the opportunity to review the manuscript for medical and scientific accuracy as well as intellectual property considerations.

We thank Dr. Alvaro Aranda (Hospital Auxilio Mutuo, San Juan, Puerto Rico) for his scientific and clinical contributions during the drafting of this manuscript. We also thank Dr. Ruth B Murray (OPC, UK) who has substantially contributed to the presentation of information in this article, provided significant intellectual input to the manuscript and final approval of the version to be published. She has agreed to be accountable for all aspects of the work in ensuring that questions related to the accuracy or integrity of any part of the work are appropriately investigated and resolved. Dr. Murray is the founder and director of Medscript Ltd., a company that provided writing and editorial support for APEX COPD publications.

To see this article online, please go to: http://jabfm.org/content/ 34/1/22.full.

\section{References}

1. Pauwels RA, Buist AS, Calverley PM, Jenkins CR, Hurd SS. Global strategy for the diagnosis, management, and prevention of chronic obstructive pulmonary disease. NHLBI/WHO Global Initiative for Chronic Obstructive Lung Disease (GOLD) Workshop summary. Am J Respir Crit Care Med 2001;163:1256-76.

2. Global Initiative for Chronic Obstructive Lung Disease. Global strategy for the diagnosis, managment, and prevention of chronic obstructive pulmonary disease. 2020 report. 2020. Available from: https://goldcopd.org/wp-content/uploads/2019/11/ GOLD-2020-REPORT-ver1.0wms.pdf.

3. Tinkelman DG, Price DB, Nordyke RJ, Halbert RJ. Misdiagnosis of COPD and asthma in primary care patients 40 years of age and over. J Asthma 2006;43:75-80.

4. Berry CE, Yawn BP. COPD overdiagnosis, underdiagnosis, and treatment. Chronic Obstr Pulm Dis Miami Dis 2016;3:491-7.

5. Diab N, Gershon AS, Sin DD, et al. Underdiagnosis and overdiagnosis of chronic obstructive pulmonary disease. Am J Respir Crit Care Med 2018;198:1130-9.

6. Ferguson GT, Enright PL, Buist AS, Higgins MW. Office spirometry for lung health assessment in adults: 
a consensus statement from the National Lung Health Education Program. Chest 2000;117:1146-61.

7. Yawn BP, Wollan PC, Textor KB, Yawn RA. Primary care physicians', nurse practitioners' and physician assistants' knowledge, attitudes and beliefs regarding COPD: 2007 to 2014. Chronic Obstr Pulm Dis Miami Dis 2016;3:628-35.

8. NIH launches new campaign aimed at increasing awareness, early diagnosis and treatment for COPD. 2007. Available from: https://www.nih.gov/newsevents/news-releases/nih-launches-new-campaignaimed-increasing-awareness-early-diagnosis-treatmentcopd.

9. CDC, National Centre for Chronic Disease Prevention \& Health Promotion; Division of adult and community health. Public Health Strategic Framework for COPD Prevention. 2011. Available from: https://www.cdc.gov/copd/pdfs/framework_ for_copd_prevention.pdf.

10. Perez X, Wisnivesky JP, Lurslurchachai L, Kleinman LC, Kronish IM. Barriers to adherence to COPD guidelines among primary care providers. Respir Med 2012;106:374-81.

11. Foster JA, Yawn BP, Maziar A, Jenkins T, Rennard SI, Casebeer L. Enhancing COPD management in primary care settings. MedGenMed 2007;9:24.

12. Yawn BP, Suissa S, Rossi A. Appropriate use of inhaled corticosteroids in COPD: the candidates for safe withdrawal. NPJ Prim Care Respir Med 2016;26:16068.

13. Plaza V, Giner J, Curto E, et al. Assessing adherence by combining the test of adherence to inhalers with pharmacy refill records. J Investig Allergol Clin Immunol. 2019;31:0.

14. Belleudi V, Di Martino M, Cascini S, et al. The impact of adherence to inhaled drugs on 5-year survival in COPD patients: a time dependent approach. Pharmacoepidemiol Drug Saf 2016;25: 1295-304.

15. COPD.NIH.gov. COPD national action plan. 2017. Available from: https://www.nhlbi.nih.gov/
sites/default/files/media/docs/COPD\%20National \%20Action\%20Plan\%20508_0.pdf.

16. Gliklich RE, Dreyer NA, Leavy MB, eds. Registries for evaluating patient outcomes: a user's guide. 3rd ed. Rockville, MD: Agency for Healthcare Research and Quality; 2014.

17. Nelson EC, Dixon-Woods M, Batalden PB, et al. Patient focused registries can improve health, care, and science. BMJ 2016;354:i3319.

18. Blonde L, Khunti K, Harris SB, Meizinger C, Skolnik NS. Interpretation and impact of realworld clinical data for the practicing clinician. Adv Ther 2018;35:1763-74.

19. Regan EA, Hokanson JE, Murphy JR, et al. Genetic epidemiology of COPD (COPDGene) study design. COPD 2010;7:32-43.

20. COPD Patient-Powered Research Network (PPRN). COPD PPRN: why you should enroll. Available from: https://www.copdfoundation.org/Research/COPDPatient-Powered-Research-Network/COPD-PPRNWhy-you-should-enroll.aspx.

21. Edwards CL, Kaplan AG, Yawn BP, et al. Development of the Advancing the Patient Experience in COPD registry: a modified Delphi study. Chronic Obstr Pulm Dis 2020; Published online November 24, 2020.

22. Soler-Cataluña JJ, Marzo M, Catalan P, Miralles C, Alcazar B, Miravitlles M. Validation of clinical control in COPD as a new tool for optimizing treatment. COPD 2018;13:3719-31.

23. Tudur Smith C, Marcucci M, Nolan SJ, et al. Individual participant data meta-analyses compared with meta-analyses based on aggregate data. Cochrane Database Syst Rev 2016;9:MR000007.

24. Williams WG. Uses and limitations of registry and academic databases. Semin Thorac Cardiovasc Surg Pediatr Card Surg Annu 2010;13:66-70.

25. Price D, Smith P, Hellings P, et al. Current controversies and challenges in allergic rhinitis management. Expert Rev Clin Immunol 2015;11: 1205-17. 


\section{Appendix}

\section{Patients: Rules of Exclusion}

Patients found to have 1 or more exclusion criteria after they have consented for the study will be left in the study as there is no intervention and they are at no increased medical risk to continue. This decision was made in recognition of the fact that documentation concerning hospice care and cancer treatments may not always be available in primary care electronic health record (EHR).

\section{Primary Care Clinic Number}

The current primary care clinic (PCC) sizes range from 1200 to 1900 patients. Each PCC is estimated to include 102 patients with chronic obstructive pulmonary disease (COPD; based on 1500 patients per PCC and a $6.8 \%$ COPD prevalence), of which $60 \%$ (ie, 61 patients) are predicted to be included in the Advancing Patient Experience (APEX) in COPD registry. Therefore, 50 participating primary care clinicians will be required to enroll 3000 COPD patients over a 4-year period. At least $50 \%$ of recruited patients will have a minimum of 2 years of prospective data and the majority at least 1 year.

\section{Patient Recruitment}

Multiple methods will be used to recruit patients and minimize attrition rates. The registry will focus on recruitment through information provided to patients in the PCC office, supplemented with invitational/opt out letter directing people to the study website where they can self consent. Follow-up phone calls (by DARTNet staff) for patients signing an interest card in the PCC office will also be used.

\section{Data Collection}

Patient reported information/patient reported outcomes (PRI/PROs) to be collected will pertain to any data the patient has access to and can accurately relay via online survey. Questionnaires will be collected using Patient Engaged Electronic Reporting System and will be compatible with multiple devices to maximize ease of data collection. Patients will be sent 3 reminder messages 48 hours apart as a reminder to complete questionnaires.

Three PRI/PRO questionnaires will be used.

1. Baseline questionnaire to include a broad set of questions including historical information related to exposures that increase the risk of COPD, family history, COPD control, quality of life, and medication preferences.

2. Annual questionnaire to update baseline information which can change over time such as exacerbations, quality of life and medication preference.

3. Short pre-clinic visit questionnaire designed to focus on data of use for clinical assessment; to be completed at least 30 days from any previous data collection.

\section{APEX COPD Organizational Structure and Responsibilities}

\section{Optimum Patient Care}

Optimum Patient Care (OPC) Global specializes in delivering medical research and services to improve the diagnosis, treatment, and care of chronic diseases within primary care. OPC has a proven track record in delivering primary care research, clinic support services, customized data sets for academic research as IG-compliant data extractions and delivering global registry projects. It acts as data custodians of the APEX COPD database, but each site/patient continues to own patient-level data contributed.

\section{Respiratory Effectiveness Group}

The Respiratory Effectiveness Group (REG) is a not-for-profit investigator-led academic initiative comprising over 420 respiratory/allergy experts globally collaborating on delivering independent, academic real-life and comparative effectiveness research in respiratory medicine. The REG initiative focuses on establishing best practice methodologies and standards, with the objective of raising the quality and profile of real-life research for all stakeholders including patients, clinicians, industry, and guideline/regulatory bodies. REG provides ethical and scientific review of APEX in COPD research study proposals.

\section{Anonymised Data Ethics \& Protocol Transparency Committee}

The Anonymised Data Ethics \& Protocol Transparency (ADEPT) committee is an independent body of experts commissioned by REG to govern the standard of research conducted on internationally renowned databases. It comprises scientists with statistical and epidemiologic experience, members with specific database related expertise, and independent clinical experts. Its function within the APEX COPD registry is to provide ethical governance and approval of study protocols requiring the APEX COPD registry data.

\section{American Academy of Family Physicians}

The American Academy of Family Physicians is one of the largest medical organizations in the United States, with 134,600 members in 50 states. Its aim is to promote and maintain high-quality standards for family doctors who are providing continuing comprehensive health care to the public. American Academy of Family Physicians provides ethical approval of APEX in COPD registry research projects and is represented on the APEX COPD Steering Committee.

\section{APEX COPD Steering Committee}

The regulation of the registry is under the jurisdiction of the APEX COPD steering committee. The steering committee determines the scientific and research merits of any research proposal that accesses and/or uses anonymous data from the registry. 


\section{DARTNet Institute}

DI is a not-for-profit 501(c)3 research institute and collaborative of US practice-based research networks. DI coordinates and supports research, quality improvement and safety activities across partner research networks through the aggregation, analysis, and standardization of EHR data, and are working to build a national collection of EHR data, claims data and PRI/PRO data. DI is a recognized Patient Safety Organization (PSO) and has significant registry experience. DI is running the APEX COPD registry in the US, including handling all practice recruitment, PRO data collection, EHR data standardization, clinical decision support development and delivery and obtaining regulatory approvals. DI is an active partner with OPC in all phases of the project.

\section{Appendix Reference}

1. Edwards CL, Kaplan AG, Yawn BP, et al. Development of the Advancing the Patient Experience in COPD registry: a modified Delphi study. Chronic Obstr Pulm Dis 2020; Published online November 24, 2020. 
Appendix Table 1. APEX COPD Registry Variables Agreed by Delphi Consensus

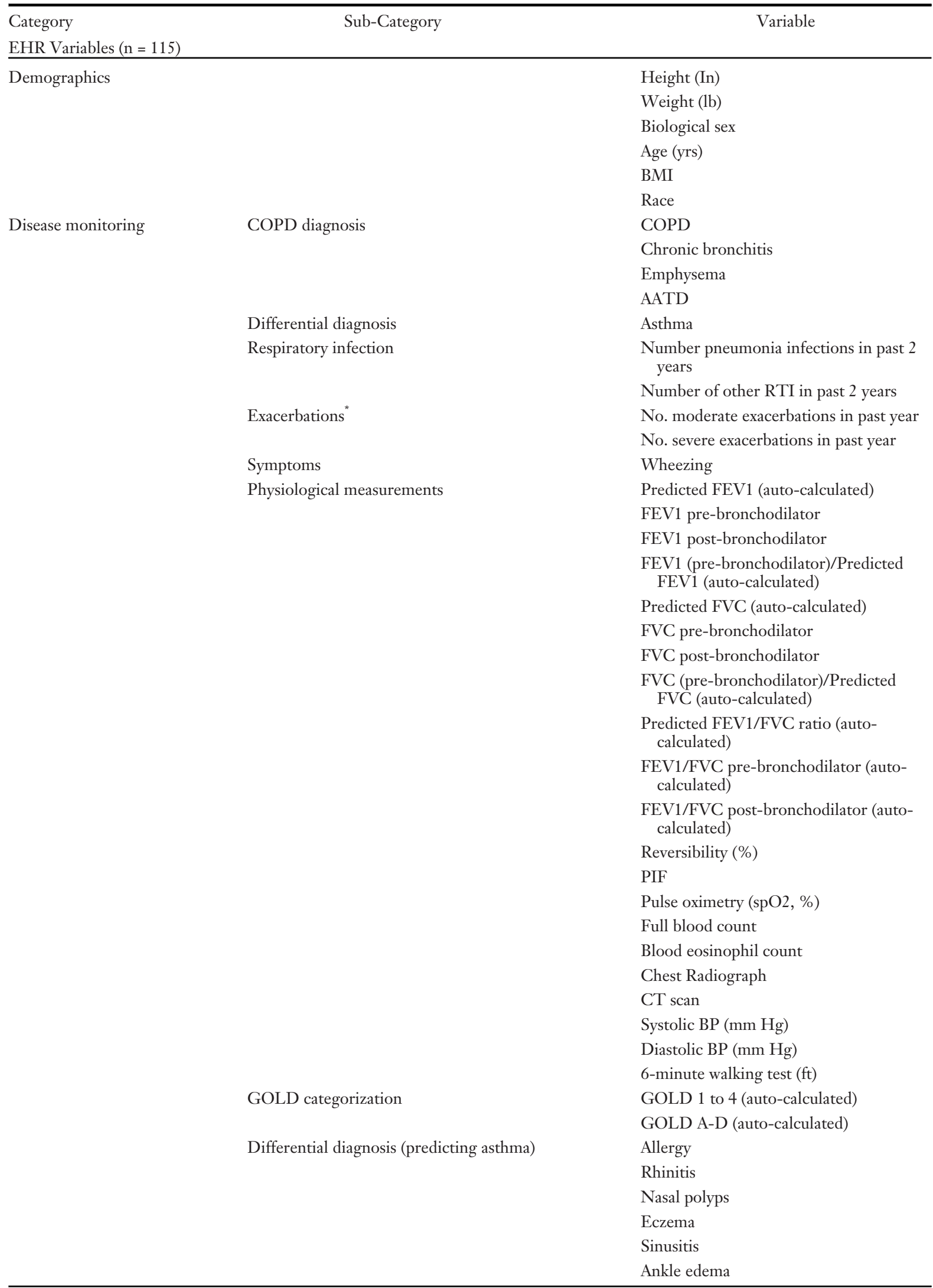

Continued 
Appendix Table 1. Continued

\begin{tabular}{|c|c|c|}
\hline Category & Sub-Category & Variable \\
\hline$\underline{\text { EHR Variab }}$ & & \\
\hline & Differential diagnosis (predicting heart & Echo cardiogram \\
\hline & failure) & Elevated B-type natriuretic peptide \\
\hline & Differential diagnosis (predicting lung & Weight loss \\
\hline & cancer) & Hemoptysis \\
\hline & Other diff. diagnosis & Pneumonia \\
\hline & Co-morbidities (cardiovascular) & Angina pectoris/Heart disease/CHD \\
\hline & & Heart failure \\
\hline & & Stroke \\
\hline & & Hypertensive disease \\
\hline & Co-morbidities (pulmonary) & Lung cancer \\
\hline & & Obstructive sleep apnea \\
\hline & & Hypoxemia \\
\hline & Co-morbidities (endocrinological) & Diabetes mellitus \\
\hline & & Osteoporosis \\
\hline & & Osteoarthritis \\
\hline & & Metabolic syndrome \\
\hline & Co-morbidities (mental) & Depression \\
\hline & & Anxiety \\
\hline & Co-morbidities (other) & GERD \\
\hline & & Anemia \\
\hline Treatment & Bronchodilators & SABA \\
\hline & & LABA \\
\hline & & SAMA \\
\hline & & LAMA \\
\hline & Steroids & ICS \\
\hline & & OCS \\
\hline & Combinations & SABA/SAMA \\
\hline & & LABA/LAMA \\
\hline & & ICS/LAMA \\
\hline & & ICS/LABA \\
\hline & & ICS/LAMA/LABA \\
\hline & Co-morbidity treatments & Heart failure \\
\hline & & Diabetes \\
\hline & & Osteoporosis \\
\hline & & Asthma \\
\hline & Other treatments & Antibiotics \\
\hline & & Macrolides \\
\hline & & PDE inhibitors \\
\hline & & Methylxanthines (eg, theophylline) \\
\hline & & LTRA \\
\hline & & Diuretics \\
\hline & & Mucolytics \\
\hline & Smoking & Smoking status \\
\hline & & How many years has patient smoked? \\
\hline & & Pack years \\
\hline & Vaccinations & Influenza \\
\hline & & Pneumococcal (both) \\
\hline & Smoking cessation & Smoking cessation advice given \\
\hline & & Referral to stop-smoking clinic/advisor \\
\hline
\end{tabular}

Continued 
Appendix Table 1. Continued

Surgery

Specialist referral

Other therapies

$\mathrm{PRI} / \mathrm{PRO}$ variables $(\mathrm{n}=34)$

Disease monitoring

Treatment
Differential diagnosis

Respiratory infections

Exacerbations*

Health status (QoL)

Risk factors

Co-morbidities (mental)

Inhaler management

Treatment side effects

Smoking
Nicotine replacement therapy

Drug therapy (eg, bupropion)

Bullectomy

Lung Volume Reduction Surgery (LVRS)

Lung volume reduction coil

Endobronchial Valve (EBV)

Lung transplant

Chest wall vibration

Palliative care

Physiotherapist

Occupational therapist

Speech therapist

Clinical psychiatrist

Clinical psychologist

Dietitian

Exercise physiologist

Chest physician

Pulmonary rehabilitation (PR)

Oxygen therapy

Home nebuliser

Ventilatory support

Asthma

No. of pneumonia infections in past 2 years

No. of other RTI in past 2 years

No. of moderate exacerbations in past year

No. of severe exacerbations in past year Modified MRC Dyspnea Scale

COPD Assessment Test

Childhood respiratory infections

Occupational exposure

Tobacco exposure

Age of onset of respiratory symptoms yrs)

Family history of COPD

Depression

Anxiety

Spacer with MDI

Inhaler technique training

Inhaler use/adherence

Test of Adherence to Inhalers (TAI) questionnaire

Inhaler satisfaction

Oral

Physiological

Smoking status

Date ceased smoking (if applicable)

How many cigarettes smoked per day? 


\begin{tabular}{|c|c|c|}
\hline Category & Sub-Category & Variable \\
\hline \multicolumn{3}{|c|}{ EHR Variables $(\mathrm{n}=115)$} \\
\hline & & How many years has patient smoked? \\
\hline & & Uses e-cigarette? \\
\hline & Vaccinations & Influenza \\
\hline & Smoking cessation & Desire to quit smoking \\
\hline & & Tried to quit in the past \\
\hline & & Smoking cessation advice given \\
\hline & Education \& self- management & COPD education \\
\hline & & COPD self-management plan \\
\hline & & $\begin{array}{l}\text { Patient's use of COPD self-management } \\
\text { plan }\end{array}$ \\
\hline & Other therapies & Pulmonary rehabilitation \\
\hline \multicolumn{3}{|l|}{ PoC Variables $(\mathrm{n}=5)$} \\
\hline \multirow[t]{4}{*}{ Disease monitoring } & Exacerbations $^{*}$ & $\begin{array}{l}\text { No. of moderate exacerbations in past } \\
\text { year }\end{array}$ \\
\hline & & No. severe exacerbations in past year \\
\hline & Physiological measures & FEV1 post-bronchodilator \\
\hline & & FVC post-bronchodilator \\
\hline Treatment & Inhaler management & Inhaler technique assessment \\
\hline
\end{tabular}

Modified from Edwards et al, $2020^{1}$.

*COPD exacerbations were defined as an explicit exacerbation (coded in a patient EMR), OR a COPD code (which included COPD, chronic bronchitis and emphysema) alongside an antibiotic and/or OCS prescription, OR an acute bronchitis, LRTI, influenza, asthma, bronchiectasis or general lower respiratory condition code alongside an OCS and/or antibiotic prescription.

AATD, Alpha-1 antitrypsin deficiency; Atb, antibiotic; BMI, body mass index; BP, blood pressure; CHD, coronary heart disease; COPD, Chronic Obstructive Pulmonary Disease; FEV1, forced expiratory volume in one second; FVC, forced vital capacity; GERD, gastroesophageal reflux disease; GOLD, Global initiative for chronic Obstructive Lung Disease; ICS, inhaled corticosteroid; LABA, long-acting $\beta 2$-agoinist; LAMA, long-acting muscarinic antagonist; LTRA, leukotriene receptor antagonist; MDI, metered dose inhaler; MRC, Medical Research Council; OCS, oral corticosteroid; PDE, phosphodiesterase; PIF, peak inspiratory flow rate; RTI, respiratory tract infection; SABA, short-acting $\beta 2$-agonist; SAMA, short-acting muscarinic antagonist; CT scan, computerized tomography scan.

Appendix Table 2. APEX COPD Steering Committee Members and Delphi Panel

\begin{tabular}{lll}
\hline David B. Price, FRCGP & Research, primary care & UK/Singapore \\
Barbara P. Yawn, MD, MSc, MSPH, FAAFP & Research, primary care & USA \\
Janwillem W.H. Kocks, MD, PhD & Research, primary care & Netherlands \\
Wilson D. Pace, MD & Research, primary care & USA \\
Ku-Lang Chang, MD, FAAFP, MRO & Primary care & USA \\
Chester Fox, MD, FAAFP & Primary care & USA \\
Barry Make, MD & Specialist & USA \\
Alan Kaplan, MD, CCFP(EM), FCFP & Primary care, specialist & Canada \\
Neil Skolnik, MD & Primary care & USA \\
MeiLan K. Han, MD, MSc & Specialist & USA \\
Alvaro Aranda, MD & Specialist & Puerto Rico \\
Gokul Gopalan, MD, MPH & Research & USA \\
Asif Shaikh, MD, MPH & Research & USA \\
Cathy D. Mahle, PhD, MBA & Research & USA \\
\hline
\end{tabular}

APEX, Advancing the Patient Experience; COPD, chronic obstructive pulmonary disease. 